Ageing E Society 38, 2018, 1817-1842. (C) Cambridge University Press 2017

This is an Open Access article, distributed under the terms of the Creative Commons

Attribution licence (http://creativecommons.org/licenses/by/4.0/), which permits unrestricted re-use, distribution, and reproduction in any medium, provided the original work is properly cited.

doi:10.1017/So144686X1700029o

\title{
'If they don't use it, they lose it': how organisational structures and practices shape residents' physical movement in care home settings
}

\author{
REBECCA J. HAWKINS*, ARVIN PRASHAR $\uparrow$, \\ ADELAIDE LUSAMBILI $\dagger$, DAVID R. ELLARD \\ and MARY GODFREY* on behalf of the REACH Programme Team
}

\begin{abstract}
Older people living in long-term facilities (nursing and residential homes providing 24-hour care) spend the majority of their time inactive, despite the known health and wellbeing benefits of physical activity and reduced time spent sedentary. In order to successfully embed interventions that aim to increase physical activity or reduce sedentary behaviour, it is necessary to understand the features of the care environment that influence residents' routine patterns of movement. Drawing on an organisational perspective, this paper explores the structures and mechanisms that shaped different care practices concerning residents' movement in two contrasting care homes in the north of England. This study adopted an ethnographic approach, using a combination of qualitative observations, informal conversations and interviews. A grounded theory approach to data analysis was adopted. The findings illustrate the importance of translating espoused values of care into tangible and acceptable care practices, systems of management, staff training and development, and the use of care planning in residents' routine patterns of movement. Understanding how organisational factors shape routine movement among care home residents will help inform the development of embedded and sustainable interventions that enhance physical activity and reduce sedentary behaviour. This study is part of a wider programme of research developing and testing a complex intervention, embedded within routine care, to reduce sedentary behaviour among care home residents.
\end{abstract}

* Academic Unit of Elderly Care and Rehabilitation, Leeds Institute of Health Sciences, Faculty of Medicine and Health, University of Leeds, UK.

$\dagger$ Academic Unit of Elderly Care and Rehabilitation, Bradford Institute for Health Research, Bradford Teaching Hospitals NHS Foundation Trust, UK.

\$ Warwick Clinical Trials Unit, Division of Health Sciences, Warwick Medical School, University of Warwick, Coventry, UK. 
1818 Rebecca J. Hawkins et al.

$\boldsymbol{K E Y} \boldsymbol{W O R D S}$ - residential care, nursing home, care environment, organisational culture, physical activity, sedentary behaviour, qualitative, ethnography.

\section{Introduction}

Older people living in permanent, 24-hour, residential and nursing home settings (care homes) spend most of their time inactive (Reid et al. 2013; Sackley et al. 20o6; Van Alphen et al. 2016). Studies from the United Kingdom (UK), The Netherlands and Australia have highlighted the need to further investigate appropriate ways of increasing physical activity and reducing periods of time spent sedentary (Reid et al. 2013; Sackley et al. 2006; Van Alphen et al. 2016).

There is an established body of evidence concerning the benefits of physical activity on physical and mental wellbeing. Among older people increased levels of physical activity can help improve or maintain physical, functional and emotional health, and protect against certain conditions (Baum et al. 2003; Blake et al. 2009; Bruunsgaard et al. 2004; Potter et al. 2011; Windle et al. 2010). Older people may derive benefit, including social benefit, from increasing their levels of physical activity (Smalbrugge et al. 20o6). In addition, there is growing evidence of the impact of sedentary behaviour, independent of physical activity, on mortality, physical function, mobility and wellbeing across the lifespan (Katzmarzyk 2010; Matei et al. 2015; Owen et al. 2010; Sardinha et al. 2015 ; Thorp et al. 2011 ).

A range of physical activity and rehabilitation interventions have been introduced and evaluated in care homes for older people in Europe, North America and Australasia (Crocker et al. 2013; Paw et al. 2008). Systematic reviews highlight that many of these interventions were shortterm, resource-intensive, excluded those with complex needs and that any gains tended not to be sustained (Crocker et al. 2013; Paw et al. 2008). There is a lack of interventions aimed at increasing routine daily movement and reducing the time care home residents spend sedentary.

In order to increase the physical activity of residents and reduce time spent sedentary, it is necessary that interventions are embedded in routine practice, and that care home staff are involved in developing and delivering necessary change (Kerse et al. 20o8). This requires increased understanding of the contextual and organisational factors that shape care practice and residents' existing patterns of movement. Despite the growing number of interventions being introduced, however, there is a lack of research into the social, organisational and environmental factors that shape sedentary behaviour and physical inactivity among older people living in residential care (Reid et al. 2013; Van Alphen et al. 2016). 
This study is located within a programme of research based in the UK aiming to develop and test a complex intervention, embedded within routine care, to reduce the amount of time care home residents are sedentary (REACH: Research Exploring physical Activity in Care Homes). Focusing on the organisational features of the care environment, this paper explores residents' routine patterns of movement in two care homes, Hebble House and Bournville, and the organisational values, structures, mechanisms and processes that shaped these patterns. Other features of the care environment that shape residents' routine patterns of movement will be reported elsewhere.

\section{Study context}

This study took place in care homes in the north of England. In the UK there exists a mixed economy of care home provision, including forprofit, third-sector and local authority providers, although the for-profit sector dominates.

Providers range in size from large corporations with multiple homes to small, family-run single-home businesses. Roughly two-thirds of residents receive some financial support from local authorities based on a means test and assessment of their need for 24-hour care; the remainder fund themselves (Killett et al. 2015). Currently, there are approximately 235,799 residential care beds and 224,843 nursing home beds for adults in England (Care Quality Commission 2016). Care home providers are under increasing pressure relating to the recruitment and retention of staff, and financial problems, which have resulted in care home closures (Care Quality Commission 2016).

\section{Background}

The care environment and the role of the organisation in shaping behaviour

The care environment of a care home is comprised of the dynamic interaction between features at the organisational, physical, social and individual levels (Moore 1999). The interaction between physical features (e.g. the organisation of space), social processes (interactions between staff, residents and others), individual factors (e.g. the needs of older people and their transition into the care setting) and organisational aspects (the organisation and delivery of care) are known to shape older people's lives and routines in care home settings (Moore 1999).

Important in understanding this link between the care environment and what residents do is the theory that the interplay between the person and 
their environment shapes action and interaction in that space (Gubrium 1978; Moore 1999, 2014; Rowles and Bernard 2013). The importance of congruence between action and place has also been acknowledged (Blumer 1986; Moore 1999). In terms of understanding action and interaction within the context of a care home, therefore, authors have adopted approaches that focus on examining the care environment and how it shapes what is acceptable and expected within the setting (Moore 1999).

In recent years increasing focus has been paid to the study of care homes from an organisational perspective, including researching organisational culture and how this shapes the care provided (Hughes et al. 2007; Killett et al. 2015). Organisational culture can be understood as shared group values, norms and practices that are (re) produced through organisational structures and processes, and the solving of real-life problems over time (Schein 1990). An organisation's culture may be more or less consistent across the organisation (Schein 1990), and the study of consistencies and inconsistencies are of importance when explaining how organisations work. Studying organisational culture involves exploring values, norms, artefacts, rituals, assumptions and myths, which are understood to shape decisions, actions, interactions, activities and routines within organisations (Kirkley et al. 2011 ; Schein 1990; Van Maanen and Schein 1979). Understanding how practice and routines are shaped within organisations is the starting point for making changes to improve practice (Schein 1990).

In the context of care homes, examining the dynamic between espoused organisational values and observed behaviour has been used to explore and explain features of the care provided (Hughes et al. 2007; Killett et al. 2015 ; Schein 1990). This approach has been applied in the study of the provision of person-centred care (Killett et al. 2015; Kirkley et al. 2011; Tyler and Parker 2011), meaningful activities (Harmer and Orrell 2008) and prescribing (Hughes et al. 2007).

Although multiple methodologies have been used to study organisational culture, ethnographic approaches are particularly useful as they allow the researcher to conduct detailed observations of the everyday life of an organisation, as well as providing a means to probe beyond the surface to explore values, assumptions and norms in interviews (Schein $1990)$.

The notion of the care environment, and the interplay between environment and action, informed the conceptual framework adopted in this study. In developing our understanding of the organisational element of our conceptual framework, we drew on literature from the study of organisational culture to explore and explain care practices. 


\section{Methodology and methods}

An ethnographic approach was adopted, using a combination of qualitative observations and interviews, to examine patterns of action and interaction within care home settings and participants' own perspectives (Atkinson et al. 2001).

Four care homes were purposively sampled for variation in relation to features that shape the structure and delivery of care provided: ownership, number of residents, type of care (residential or nursing care) and specialist provision. For the purposes of this paper, we draw on data from two care homes, Hebble House and Bournville. The similarities and differences between them provide an interesting comparison with which to present the findings from the analysis (see Table 1 ).

Ethnographic observations (Atkinson et al. 2001; Spradley 1980) were conducted within the communal spaces on approximately two days per week over a period of approximately four months in each home by researchers (RJH, AP, AL). Observations were conducted on different days of the week, in different internal and external areas of the homes, and encompassed daytime, evenings and weekends. An observation guide was produced drawing on the conceptual framework and initial observations, to support on-going focusing of the observations. The guide included prompts to observe domains such as care practices, daily routines, what work is prioritised, the organisation and delivery of care, space and use of space, interactions between staff and between staff and residents. Researchers engaged residents, staff members and visitors in ethnographic conversations (Spradley 1980) to explore the meanings people gave to events taking place. These conversations were particularly helpful for involving those residents with dementia and busy staff who did not have time to participate in formal qualitative interviews. Detailed field notes were produced to capture the observations.

A purposive sample of staff occupying varied roles were approached from each home to take part in interviews, including care assistants, activity coordinators, domestic staff, senior care staff and managers. Staff differed in the nature of their involvement in the organisation and delivery of care and routine activities. Residents were sampled for interview based on diversity of their physical and cognitive capabilities, daily routines and level of physical movement. Their relatives were also approached for interview.

Fifty-five qualitative interviews were undertaken across the four participating care homes towards the end of the period of observation, and aimed to help clarify and further explore findings from the observation work (22 interviews with staff members, 16 interviews with residents and 17 interviews with relatives). At Hebble House, interviews were conducted with four 
T A B L E 1. Summary of Hebble House and Bournville

\begin{tabular}{|c|c|c|c|c|}
\hline Ownership & Registration details & Staff profile & $\begin{array}{l}\text { Resident profile (at } \\
\text { time of observations) }\end{array}$ & Illustrative pen portrait of a care home resident \\
\hline $\begin{array}{l}\text { Hebble House: } \\
\text { Local authority }\end{array}$ & $\begin{array}{l}\text { Residential. } \\
\text { Registered to } \\
\text { provide care for } 35 \\
\text { residents. } \\
\text { Specialisms: } \\
\text { dementia, caring for } \\
\text { adults over } 65 \text { years. }\end{array}$ & $\begin{array}{l}\text { Approximately } 65 \\
\text { members of staff. } \\
\text { Activity co-ordinator } \\
\text { absent during research. } \\
\text { Small number of staff } \\
\text { have completed a course } \\
\text { on purposeful exercise. } \\
\text { Majority have com- } \\
\text { pleted training on } \\
\text { dementia. }\end{array}$ & $\begin{array}{l}\text { Majority of residents } \\
\text { aged } 80+\text { and } \\
\text { female. } \\
\text { Residents varied in } \\
\text { terms of their phys- } \\
\text { ical abilities. } \\
\text { All residents had a } \\
\text { diagnosis of demen- } \\
\text { tia (mild, moderate } \\
\text { or late-stage } \\
\text { dementia). }\end{array}$ & $\begin{array}{l}\text { Joy is in her mid-seventies. She grew up in the local } \\
\text { area, but lived away from the area for much of her } \\
\text { adult life. Joy was very active when she was in her } \\
\text { twenties and thirties, but then experienced a long } \\
\text { and gradual decline in her physical abilities, which } \\
\text { was exacerbated by the stroke she had in her fifties. } \\
\text { Joy has had several strokes since, which have had an } \\
\text { impact on both her physical and cognitive abilities, } \\
\text { and has vascular dementia. She has lived at Hebble } \\
\text { House for four years. Her daughter visits her every } \\
\text { fortnight. Joy is a friendly and generally jovial char- } \\
\text { acter and is often heard laughing. Joy will initiate and } \\
\text { engage in the odd conversation with others when she } \\
\text { is sat in a lounge area. Joy walks very slowly and with } \\
\text { great effort using a Zimmer frame, and often } \\
\text { requires verbal encouragement and instruction from } \\
\text { staff members to mobilise. }\end{array}$ \\
\hline
\end{tabular}


TABLE 1 . (Cont.)

\begin{tabular}{|c|c|c|c|c|}
\hline Ownership & Registration details & Staff profile & $\begin{array}{l}\text { Resident profile (at } \\
\text { time of observations) }\end{array}$ & Illustrative pen portrait of a care home resident \\
\hline $\begin{array}{l}\text { Bournville: } \\
\text { Private; part of a } \\
\text { large, international } \\
\text { company }\end{array}$ & $\begin{array}{l}\text { Residential. } \\
\text { Registered to } \\
\text { provide care to } 30 \\
\text { residents. } \\
\text { Specialism: caring } \\
\text { for adults over } 65 \\
\text { years, physical } \\
\text { disabilities. }\end{array}$ & $\begin{array}{l}\text { Approximately } 30 \\
\text { members of staff. } \\
\\
\text { Recently appointed } \\
\text { activities co-ordinator. }\end{array}$ & $\begin{array}{l}\text { Residents varied in } \\
\text { terms of their phys- } \\
\text { ical abilities. } \\
\text { A small number of } \\
\text { residents had a diag- } \\
\text { nosis of mild } \\
\text { dementia. }\end{array}$ & $\begin{array}{l}\text { Poppy, could be described as being fairly typical of } \\
\text { her peers living at Bournville care home. } \\
\text { Poppy (who is in her nineties) had moved to } \\
\text { Bournville care home approximately two years } \\
\text { earlier. She had been very active within her local } \\
\text { community before moving into residential care. } \\
\text { During the period of fieldwork Poppy spent most of } \\
\text { the time oscillating from her room to the lounges, } \\
\text { and when in communal spaces would sit quietly by } \\
\text { herself. Her capacity to communicate was limited, as } \\
\text { a result of dementia, so she spoke in a fairly slow and } \\
\text { deliberate fashion. She infrequently spoke to the } \\
\text { other residents, other than at mealtimes, and the } \\
\text { conversations often seemed quite short and stilted. } \\
\text { Her family would visit her each week. Poppy used a } \\
\text { Zimmer frame to move from space to space, and she } \\
\text { would usually sit adjacent to the television set, } \\
\text { although did not watch television. Despite the phys- } \\
\text { ical effort involved in moving about, Poppy main- } \\
\text { tained the determination to do so. She did not } \\
\text { participate in any activities, although few were pro- } \\
\text { vided during the period of observation. On the } \\
\text { occasions when Poppy did attempt to take part in } \\
\text { activities there was often insufficient support to } \\
\text { enable her to engage successfully. }\end{array}$ \\
\hline
\end{tabular}


members of staff (the manager, assistant manager, a senior care assistant and a care assistant), as well as with two residents and two relatives. At Bournville, interviews were conducted with four staff members (the manager, a senior care assistant and two care assistants), as well as four residents and six family members. The topic guides were informed by the observation work and the conceptual framework. The topic guide for the staff interviews included: their role, organisational aims and priorities, daily routine, how work is managed and organised, and residents' daily routines and physical activity. The topic guide allowed for flexibility by allowing researchers to draw on their observations to inform the interviews. Interviews were conducted, where possible, in a quiet private area, and were audio-recorded and transcribed verbatim. A flexible approach was taken to conducting the interviews to ensure they were as inclusive as possible.

Permission was gained from National Health Service (NHS) ethics, care home managers and, if required, key personnel within the wider care home organisation to undertake the research. Informed consent or consultee opinion was sought for focused observations (i.e. shadowing particular staff members or spending time with particular residents) and interviews. Pseudonyms (including the names of the care homes) are used throughout, and efforts have been made to remove identifiable information.

A grounded theory approach to data analysis was adopted to enable the move from describing routine patterns of movement, action and interaction to developing explanations of why such patterns existed. Analysis commenced alongside data collection, to inform the focusing of the observations and the development of the topic guide (Charmaz 2000, 2014).

The process of analysis involved each researcher (RJH, AP and AL) reading and re-reading the data and conducting open coding, paying particular attention to action and interaction (Charmaz 2000, 2014). Initially researchers coded their fieldwork notes, organising the data into meaningful units, then created written summaries of their open codes and emerging analytic ideas for discussion in regular team meetings (Charmaz and Mitchell 2001). Comparing and contrasting codes between homes generated discussion and decisions on focused codes, those most analytically significant codes that best captured the data (Charmaz 2014). A similar process was adopted for the analysis of the qualitative interviews. Focused codes included: working to support the mobility of residents; 'doing for' residents; planning to maintain mobility; actively monitoring residents' changing mobility; feeding back from practice; using care plans as repository of information; resources as a barrier to delivering activities and exercise for residents; defining the care values; explaining how care values work in practice; and barriers to enacting values in practice. Following such meetings, researchers revisited their data to reflect on the appropriateness of 
focused codes against the data-set, including across different data sources (observation and interviews) and different care homes. NVivo 8, the software program, was used to help organise and code the data, as well as to keep analytic memos.

Theoretical coding was undertaken in meetings with particular attention paid to context, processes, action and interaction, contingencies, and consequences (Charmaz 2014). The notions of the care environment and organisational culture were used as sensitising concepts at this stage to discuss and reflect upon the focused codes, the relationship between the codes and the theoretical codes. This was an iterative process, moving backwards and forwards between focused codes, data and analytic ideas captured in memos. As analysis progressed, an explanation was developed as to how different elements of the care environment, and organisational features, shaped routine behaviour, action and interaction.

\section{Findings}

Residents' general routine pattern of movement across a daily period was characterised by periods of rest and largely sedentary leisure and social activities, which were punctuated by short, routinised bursts of movement and activity centred on regenerative activities such as daily hygiene work and mealtimes. Although this general pattern was observed across all participating care homes, there were nuances and important differences between them in how this pattern was manifest. To understand such nuances, it is helpful to conceive of movement in relation to different aspects of care home life: everyday routines, organised leisure and social activities, and supporting meaningful activity.

\section{Residents' routine pattern of movement}

Movement in relation to everyday care home routine. Physical movement evident at the care homes tended to focus on carrying out tasks central to the delivery of care in the homes, for instance, maintaining residents' hygiene and organising mealtimes. This type of work was delivered in different ways in the care homes.

At Hebble House staff engaged in enabling work with regards residents' mobility during the performance of daily routines such as toileting, bedtime and mealtimes. Residents varied in the degree of support required to move around, with some able to walk independently while others required hoisting into a wheelchair and pushing. The majority of residents, however, required some form of support to walk around the home, and there was a 
consistent individually tailored approach adopted by care staff to enable such residents to mobilise. Care assistants often went to great lengths to encourage verbally, guide or physically support those who required aids or help to move. This enabling approach to routine mobilising consumed considerable time and resources. Care assistants were also observed discussing residents' changing mobility. Efforts were made to assess appropriately residents' changing needs and provide them with the correct aids and support to enable them to maintain their mobility and manage the potential risks associated with residents' mobilising.

There was also variation in the physical capabilities of residents at Bournville, with the majority requiring aids or support to walk. The enabling approach evident at Hebble House was not observed at Bournville, where the efficient and timely carrying out of routine care work was prioritised by the care team. During routine activities such as moving to the dining room for meals, residents tended to be either wheeled there or, for those who did walk independently, observed by care staff from a distance. This monitoring from a distance allowed care staff to do other tasks while reacting if required (e.g. if a resident fell). A small number of care assistants did encourage and support residents' to move, however, this was done intermittently and there was not a consistent approach to how each individual resident was supported. Despite the marked decline of one residents' mobility during fieldwork, there were no observed discussions or instances of residents' mobility being assessed.

Movement in relation to organised leisure and social activities. There was an activity co-ordinator in post at both homes. Both care homes had a timetable of activities. A few activities were explicitly aimed at physical movement (e.g. exercise classes) while the majority focused on socialising or hobbies (e.g. bingo or craft activities) that may involve a degree of movement. At both care homes the timetabled activities did not routinely take place.

At Hebble House, the activity co-ordinator was absent for the duration of the research. A few care assistants, however, did plan or spontaneously engage residents in activities, such as baking, reminiscence activities, quizzes and ball games. These activities took place intermittently over the fieldwork period, as delivering such activities required care assistants to create time away from their daily tasks. The care home manager praised those who arranged such activities for residents. Some care assistants were very skilled at tailoring activities to individual residents' capabilities. In addition to the ad hoc activities arranged by care staff, a fortnightly seated exercise class was delivered by an external provider.

At Bournville, the activity co-ordinator was new in post when fieldwork commenced and was regularly co-opted into supporting the routine delivery of care tasks, at the cost of scheduled group activities that were regularly 
cancelled. When group activities did take place, care staff did not actively support or enable residents' to participate in the activities. The activity coordinator did manage to deliver one-to-one sessions with individual residents in their rooms, and, towards the end of the research period, she successfully delivered regular seated exercise classes, which were well attended.

Movement in relation to supporting meaningful activity. Periods of rest and leisure activities initiated by residents, which tended to be characterised by residents spending time sitting in lounge areas or in their bedrooms, were also important features of residents' daily routine.

At Hebble House, residents spent the majority of time between mealtimes seated in the lounge areas resting, talking, watching television or listening to music. Care assistants were busy with various tasks during these periods, so were not consistently present in the lounge areas, and residents were sometimes left to their own devices. When care assistants were present they would be writing care plans while talking with residents, serving drinks, checking on residents or delivering an activity. Residents who enjoyed music sometimes spontaneously danced or sang, which was encouraged by care assistants, who joined in. A few independently mobile residents would spend their time walking around the care home, while those who required support to move were generally encouraged to stay seated by care staff unless they wished to move for a particular purpose (e.g. to another lounge area), in which case they would be supported. The few residents who engaged in household tasks, such as washing up and tidying, were encouraged to do so and supported by staff. During periods of good weather, residents occupying the lounges on the ground floor were encouraged to spend time outside in the garden, with those requiring help to move supported to access such spaces. There was also a small budget for activities, so care staff initiated conversations with residents regarding preferences for activities and worked to enable these to take place.

At Bournville, residents similarly spent time between meals sitting in lounge areas resting or watching television, or in their rooms. Care assistants would be engaged in a range of tasks during these periods, including responding to regular buzzers indicating residents' requests for assistance and writing care plans in a room away from residents, and so would not necessarily be present in the lounge areas. In general, care staff discouraged residents' moving during such periods and encouraged them to stay seated, due to the possible fall risk. Those residents who were able to engage in sedentary leisure activities independently, such as reading, were left to do so. There was generally little support provided to enable residents to engage in leisure and household activities if they required assistance or encouragement to do so. One resident, who had been a keen gardener, stopped 
tending to the care home gardens as it was becoming increasingly difficult for him to do so independently due to his physical condition and there was no support available to allow him to continue.

\section{How organisational factors shaped residents' routine patterns of movement}

Drawing on an organisational perspective, we now explore some of the structures and mechanisms that contributed to the shaping of these differing care practices in relation to enabling and supporting residents. In particular, we examine the espoused organisational values and how these were translated (or not) into practice through management structures and processes, staff supervision and training, and care planning (see Figure 1).

Espoused organisational values. Evident at both care homes were certain values, espoused by managers of the organisation, that encapsulated the nature of the care they aimed to provide - the ethos of the organisation.

The espoused organisational values at Hebble House and Bournville were, in many ways, very similar. At Hebble House, the care home manager, Laura, highlighted that high-quality care is concerned with empowerment, which in the context of care meant enabling residents to maintain their capabilities:

People don't see capacity, they just see the word dementia and then they think that people don't have capacity, but they still have capacity ... it's about how you enhance and keep that capacity level with them with regard to any form, any decision making about their life ... You've got to try to empower them as much as you can. (Laura, care home manager, Hebble House)

The care home manager articulated that, in practice, this entailed staff enabling residents' to make small decisions, allowing them to feel useful and supporting them to do things that they were able to do, such as performing everyday household tasks and, in particular, maintaining their physical mobility.

At Bournville, the value of supporting residents to live life to the fullest was voiced by the current manager, Clarissa, echoing that of the wider corporation. In relation to the provision of care, this involved promoting people's independence by not taking away their existing skills:

[What kind of service does this care home aim to provide?] Oh (sighs), a safe haven for people who can no longer live at home. The ability to promote independence for them, to get back to being independent and not take all their daily living skills off them ...to secure and give them support to live their life to the fullest. (Clarissa, care home manager, Bournville)

Additionally, the care home manager explained that allowing residents to live life to the fullest required that their choices and preferences with 


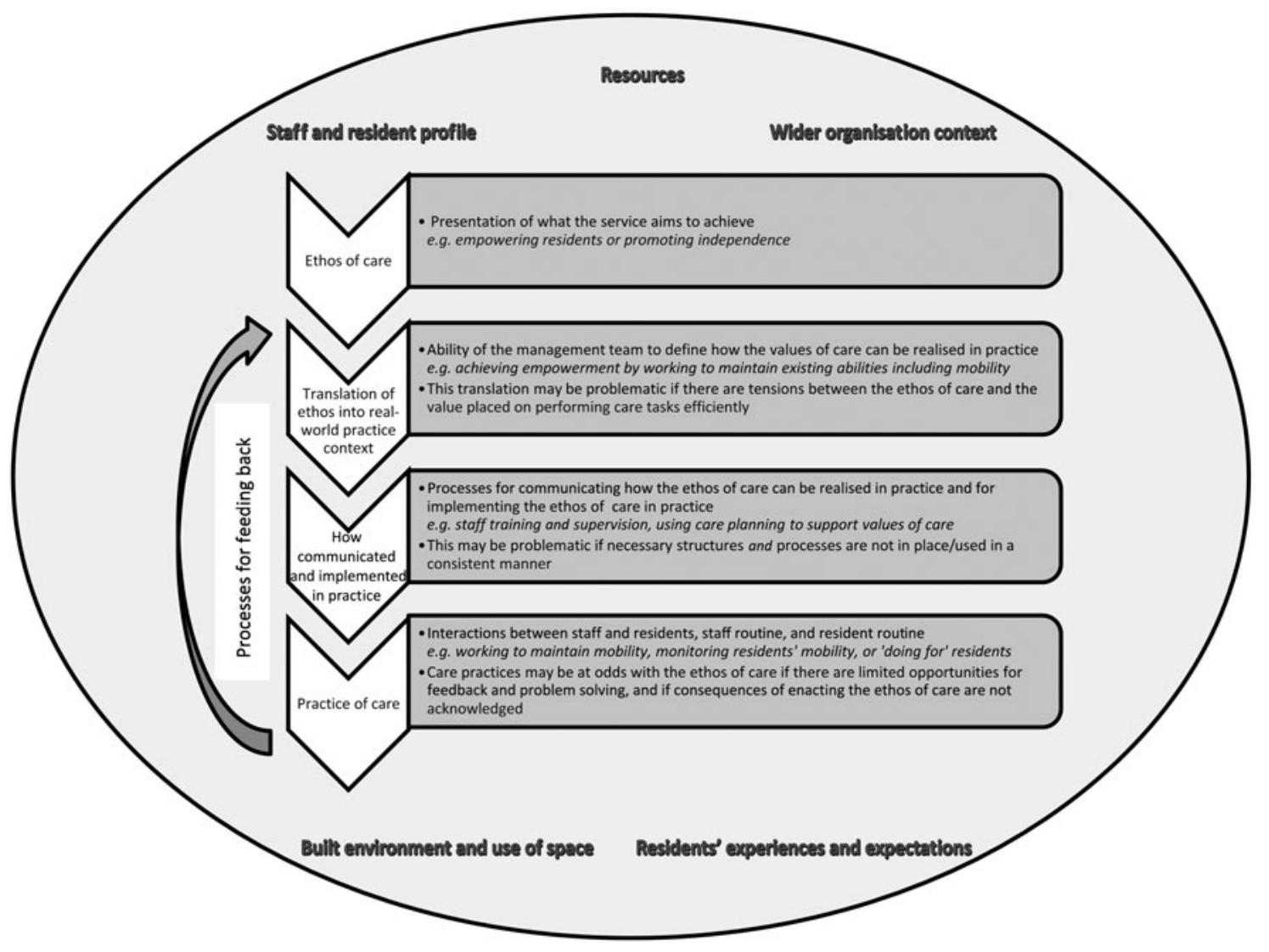

Figure 1. A grounded theory analysis of how the organisational culture of care homes can shape residents' routine patterns of movement. 
regards to leisure activities and daily routine should be promoted, and that they should feel valued:

Everything surrounds the individual and the individual's at the centre of it. It's their choice, choices and preferences that are took on-board now ... that is something I'm keen, because at the end of the day just because they come into care doesn't mean to say they have to give up all rights of their choice and preferences and their lives. (Clarissa, care home manager, Bournville)

The similarities between the espoused organisational values at the two care homes are clear, there was a claimed emphasis on working to maintain residents' abilities, treating them as people and ensuring they felt valued. There were subtle differences, however, as the espoused values of Hebble House placed more emphasis on enabling and supporting residents to maintain specific capabilities such as mobility and making small decisions, while at Bournville there was greater weight placed on choice and individual preference at a somewhat abstract level. Of interest is how such values were enacted in practice.

Management structure and processes. A consistent and coherent approach from the management team relating to how care practices could reflect the espoused organisational values; and having mechanisms in place to negotiate meaningfully and resolve issues relating to the everyday demands of care practice, were important factors in shaping the pattern of residents' movement.

At Hebble House, empowerment was translated into several discrete practical goals, the most pervasive of which was maintaining residents' ability to mobilise. The importance of supporting residents to maintain their mobility was consistently articulated by the management team:

I feel if you let people give in too early and if they don't use it, they lose it, don't they? So, and I don't like to think that they've lost it because, to me, if they can move about, they've got a lot more to look forward to ... the ones that can get up and walk about, and that are physically active, can see different things. They can be all over the [care home], they can go up and down in lift, they can go upstairs and see a different face or scenery ... And I, myself and the rest of the management team, I think, feel that it's very important to keep mobility going as long as possible. (Karen, assistant unit manager, Hebble House)

For the management team, keeping residents mobile meant working to make sure that they were able to move about and walk (perhaps with the support of walking aids) for as long as possible. Residents who were able to mobilise were thought to have a better life quality as they were less dependent on staff and they had the ability to move freely around the care home.

The message from the management team was also consistently echoed by care staff: 
...so you've just got to find ways of keeping them moving, that's the main thing is keeping them moving, we have this thing about not keeping service users in bed, they'd have to be very, very ill, you know, like care of the dying basically but if they're not on any form of the doctor says to us, you know, that they have to be in bed, get the service user up. (Tina, senior carer, Hebble House)

Members of the management team openly acknowledged that working to keep residents moving meant care assistants often spent longer carrying out routine activities, which, in the context of a sometimes hectic work environment, reinforced the value placed on such enabling work and that it was an important part of a care assistant's role.

Additionally, structures and mechanisms were in place to resolve issues that arose due to the everyday demands of care. The care home manager emphasised the importance of a reflective practice approach, and at times utilised staff meetings to reflect on and resolve problems and issues:

Staff meetings, the team meetings I'm attending, they all have, each wing has a team meeting and I've managed to attend two so far because there are issues with regard to communication at the moment. The teams aren't communicating properly so we've addressed that in two of the team meetings. I've just got two more to do. (Laura, care home manager, Hebble House)

These factors contributed to consistency amongst care staff at Hebble House regarding the importance of, and the mechanisms for, supporting residents to maintain their mobility, as well as a sense of a shared goal regarding this aspect of care.

At Bournville, despite the emphasis the care home manager placed on independence and choice, there was a lack of coherence and consistency in the priorities voiced and enacted in practice by the management and senior care staff. The management team were unable to envision how the somewhat abstract values of 'independence' and 'choice' were compatible and could be implemented in practice:

...to maintain independence I feel as a manager of the home rightly or wrongly and whether we do it intentionally I feel we take away their independence quite a lot because we do their cooking for them, we do their meals, we do the washing, we do the cleaning, you know, and they're expected to put up with that. Somebody may have been a hard worker all their lives and done all their own cleaning and washing right up to 90 to coming in and then all of a sudden you don't need to do it. We'll do all that for you. We're taking it all away. (Clarissa, care home manager, Bournville)

This contributed to the sense amongst some care staff that providing care that promoted residents' independence and choice was problematic in practice, with implications for residents' routines:

Say this morning, I encourage Hetty to come through to the lounge to sit with the other residents. 'No, I just want to stay in my own room.' And we find that ... 
Yeah that's, but that's her choice, you know, we do encourage her to come through and say, you know, 'do you want to join us for lunch?' 'No, I'll stay here.' And I said 'well, you know, we'll take you through...' and she'll 'no, no I'm alright in me room, I've got a lovely view' and I think 'bless you, you can't see half of what's in that garden', you know, to sit there in that room all day long is a sad affair really, you know. (Lydia, care assistant, Bournville)

In practice, a more task-oriented approach to caring was demonstrated in both the staff interviews and the observational data. Getting care tasks done was prioritised, so resources were channelled into achieving this goal at the expense of encouraging and facilitating physical movement:

Gail (a member of care staff) comes to speak with Vanessa (the activity co-ordinator). The gist of the conversation seems to be that she is required to help the others with care duties. Today there is supposed to be arts and crafts this morning. Vanessa walks out of the lounge. The arts and crafts session does not take place. It is not uncommon for planned leisure activities to be cancelled, and for Vanessa to help with care tasks. (Extract from fieldwork notes, Bournville)

When the manager observed care practices that she did not feel reflected the organisational values, there was an absence of appropriate mechanisms to meaningfully work to resolve them. For instance, discussions at staff meetings focused on admissions and the health status of current residents, rather than providing a forum for reflecting on how care practices could meaningfully promote residents' choice and independence.

These factors contributed to the lack of internal consistency regarding the organisational values and how these could be translated into care practices. This situation was exacerbated by staff shortages and problems of recruitment, which was emphasised in several staff interviews, leading to additional strain on staff.

Staff supervision and training. The nature of the supervision and training care staff receive are important in facilitating the communication of espoused organisational values and can be used as a mechanism for reflecting on and developing care practices. The way staff are supervised and trained shapes care practices and, therefore, the pattern of residents' movement in care homes. At both care homes there were formal supervision and training processes provided for care staff, as well as informal training and feedback provided by managers and senior care staff during usual care routines.

Laura, the care home manager at Hebble House, emphasised the importance of the supervisory process as a mechanism through which she could communicate the espoused values of care:

It's your training that you give to your staff as well, so like through your supervisions and appraisals your ethos, my ethos as a manager about the care standards that are 
expected has to come through, through the supervisions and appraisals. (Laura, care home manager, Hebble House)

The managers at Hebble House highlighted the importance of staff supervision and training, and dedicated a significant amount of time to both. Care staff also emphasised the value of training they had undertaken, including training in dementia, moving and handling, and reminiscence therapy. Maya, a care assistant, also stated that they were able to request training that they thought would be helpful.

In addition, the manager informally monitored and provided feedback to care staff members, acknowledging her responsibility to offer staff opportunities to develop:

I also monitor my staff. I am a great believer in watching staff. You can gauge a lot by just seeing how the staff are interacting with the service users. I know the staff well enough now to know my naughty staff and my good staff, and you get naughty staff wherever you are ... And it's about me being proactive as well, making my presence felt on the floor and addressing bad practice if you see it there and then. (Laura, care home manager, Hebble House)

She would highlight good and bad practice on the floor, as well as suggesting approaches and giving care staff permission to empower residents in small ways:

And it's about trying to instil into the staff that they've got to try and let them do it for themselves, you know what I mean? It's like Sophie taking Mavis round, Sophie's the laundry assistant and I said to Sophie 'take Mavis with you, let her do something'. 'Oh can I do?' 'Yeah, course you can do that', d'you know what I mean? It's just small little things isn't it? (Laura, care home manager, Hebble House)

Using staff supervision, and informal training and feedback in this way, the management team of Hebble House communicated and demonstrated how the espoused values could be delivered in practice. Providing instant practical feedback and suggestions shaped care practices and had positive implications for residents' routines.

The training and supervisory process were also discussed at Bournville, however, there was less emphasis placed on using these to communicate the espoused values and reflect on care practices. As at Hebble House, the manager also informally monitored care staff. She tended, however, to highlight poor practice and criticise staff whilst holding them accountable:

Two care assistants are hoisting a resident (Geraldine). Clarissa, the care home manager, asks one of the care assistants 'when did you do your handling and lifting (course)?' to which she replies 'ages ago'. Clarissa retorts 'it's time you did it again'. There is an uneasy atmosphere between them, as Clarissa chastises her and says pointedly 'you're not to lay hands on a resident'. Both care staff exchange 


\section{Rebecca J. Hawkins et al.}

a joke when the action has been completed, and Clarissa comments 'I think Geraldine wants to go to the toilet' in exasperation and rolls her eyes. (Extract from fieldwork notes, Bournville)

The care home manager spent a considerable amount of time on the 'shop floor'. She saw her ability to spend time with residents as one of the benefits of her role as manager. She did not believe the staff had time to sit and talk to residents as she did. Consequently, she viewed her interactions with residents as giving extra, rather than as an opportunity to demonstrate how routine care should be delivered:

So if somebody's feeling down and I can make them feel better just by sitting down, which I do, I can take the time to do that ... And if I can make somebody feel so much better just by one of my actions, that's what makes my whole role worthwhile ... The staff unfortunately can't at the minute, they can't sit down and do that but I can. And I can say 'right, no I'm not answering that telephone call, I'm going to sit with this resident for five minutes, don't disturb me' and that can happen and that's what I like. (Clarissa, care home manager, Bournville).

The disjuncture between the espoused organisational values and care practices persisted at Bournville, in part, because supervision and training were not used to provide opportunities to demonstrate and reflect upon how care staff could deliver care in ways that were compatible with choice and independence in the context of the everyday demands of care. This had implications for residents' routines and patterns of movement, as a more mechanistic approach to delivering care prevailed amongst most staff.

Care planning. At Hebble House the care plans were used to realise the goal of empowering residents through the discrete practice of maintaining their mobility during routine activities. For those residents whose mobility was thought to be at risk of declining, either due to worsening impairments or a reluctance to mobilise, plans were put in place to ensure they were provided with the correct support, be that in the form of equipment or staff, to move.

Important to the success of these plans was the input of experts with regards to the assessment and development of strategies. Importantly, they observed and worked with care staff, furthering their skills:

when we struggle [with maintaining a resident's mobility] and say, 'Right, it's time to get the moving and handling advisor in now', and so we ring [name], our moving and handling advisor, and she'll come out and she'll assess how the staff are dealing with that person. Are they safe to walk? Are they safe to mobilise? And if they're not, she makes the decision that they have to be hoisted ... If we find that they're trying to walk ... we would ring her back and we'd say, 'Little bit different this week, so and so's mobilising again, or trying to, would you just come out and have a look at her for me?' And she'd reassess her to say whether she's, you know, 
decided yeah, okay, she can walk again, and she'd say, 'Well, try her' but we'd try her with two staff, you know, so they are the ones that set what we need to do, really, yeah, and they train us on our moving and handling. (Karen, assistant unit manager, Hebble House)

Formal moving and handling support ensured that the care plans were informed by the appropriate expertise, and that staff had the opportunity to develop their own moving and handling skills.

Furthermore, care staff, in particular those who were more experienced or had worked at the home for a longer period of time, viewed maintaining residents' mobility as an important part of their role. These staff members not only worked to deliver the plans but also reflected on and provided feedback regarding how they worked in practice, as well as the shifting needs of the residents. This meant that the mobility plans were 'living' and responsive to the changing needs and mobility of residents, rather than being static documents that were revised at fixed time-points. Care assistants, in particular those with more experience, were observed engaging in discussions about particular residents' mobility if they noted a change (both in terms of an improvement or decline in mobility). The extract below was part of on-going conversations about Grace's declining mobility and referral for a moving and handling assessment:

Denise (care assistant) and Molly (care assistant) brought Grace (a resident) into the lounge area in her wheelchair. Both of them supported Grace to stand and transfer into her usual comfy seat, while they did so they verbally guided and encouraged her. Afterwards, they commented on Grace's changing mobility. They noted that she now takes very little of her own weight when she was being transferred. (Extract from fieldwork notes, Hebble House)

In addition to the formal assessment of residents' support needs were plans for those residents who were able, but reluctant to walk:

We've one service user who is very reluctant to walk, they [care assistants] set a plan where they would walk him a certain distance down the corridor every day and back, and that has to go on every day to keep him mobile, 'cos if this gentleman loses it, what has he got? ... we want to try and keep him mobile. (Karen, assistant unit manager, Hebble House)

Essential to the successful implementation of these plans was that care assistants were actively involved and supported in the monitoring and assessment process, and that this was acknowledged and acted on by senior staff:

if there's anything that needs updating in our care plans, if a service user's mobility has gone downhill and we've got them assessed and they have a Zimmer frame we try and, we write it down and we'll pass that on to the senior and we'll allow the senior to update the care plan, so that's one thing less for us to do so they'll do that for us. But I think that would be better if we had somebody else to do the paperwork, we could 
just pass it on and they could do it, and we could just concentrate on our service users and what they need. (Maya, care assistant, Hebble House)

Although Maya bemoaned the volume of paperwork care assistants had to complete, which she felt detracted from the time they could spend with residents, she viewed monitoring and assessing residents' mobility as part of her role.

The mobility plans contained within the residents' care plans at Hebble House were living documents, informed by professional expertise, and were used as a mechanism for which care staff could feedback and reflect on the changing needs of the residents to refine and adapt the plan. This allowed them to pro-actively manage risks associated with declining physical function by ensuring residents were adequately supported. These plans were used in this way primarily for those residents who had declining mobility or were reluctant to walk. Due to the manner in which they were implemented in practice, they predominately shaped residents' movement during routine activities that were predictable and 'plan-able' (e.g. walking to the dining room).

In contrast, care plans at Bournville care home were predominately used as repositories of information for monitoring individual residents rather than tools to shape daily care practices:

As Gail (a care assistant) completes a Care Plan, we talk about the process of completing them twice a day, once by day staff and again by night staff. Both Gail and the trainer explain the current system of documentation, and show me a list of codes that are used as an indicator of health status. The use of a numerical code is intended to make the data recording process for each resident easier and quicker for staff to use. (Extract from fieldwork notes, Bournville)

Care planning did not provide care staff with a means of consistently implementing care practices tailored to individual residents and was less successful at responding to their changing needs. In practice this meant that care staff sometimes supported the same resident differently with regard to supporting their mobility; this was demonstrated both in staff interviews and the observation:

A care worker wheels Belinda, a resident, to a comfy chair and tells her she'll be more comfortable sitting there, but she seems unconvinced. The care worker leaves her for a few minutes, during which time Belinda manages to stand up, turn around and be seated in the comfy chair. It is interesting to observe the response of two care workers to this incident: one of them says positively 'see Belinda, you can do it', whereas the other care worker, who had intended to help Belinda into the chair, looks aghast, perhaps fearful that Belinda may have fallen and injured herself. (Extract from fieldwork notes, Bournville)

Completing care plans that were static documents was viewed as an onerous and unpleasant chore that seemed relatively meaningless in the context of the daily delivery of care: 
At the large dining table there is a senior carer, Lianne, and a care assistant. Next to the trolley are a number of ring binders and Lianne tells me that these are individual care plans. She expresses an air of frustration on two fronts: firstly, that completing this paperwork means that the staff can't do 'other things' and secondly she points out that it can be difficult to complete the Care Plans if there is nothing much to add that constitutes new material from the previous day. Information concerning matters such as the kind of assistance that they require has generally been recorded already. She tells me that food plans tend to be recorded only for the first couple of weeks, and if things seem normal, then this is stopped unless residents start to lose weight. (Extract from fieldwork notes, Bournville)

Care plans at Bournville became repositories of information about residents that did not shape daily practice in any particular way unless the information indicated that a specific resident was unwell. They did not provide a mechanism for embedding in practice the espoused organisationalvalues, nor as a means of care staff feeding back the complexities of doing so in practice or the shifting needs of residents. In practice, therefore, care staff tended to be risk averse and discourage movement amongst residents to prevent possible falls.

\section{Discussion}

This paper focuses on how the organisational features of the care environment shaped residents' routine patterns of movement in care home settings. As far as we are aware, this is the only study to date that has examined this topic in depth.

Found to be of importance to residents' routine movement was the ability of the management team to translate the often abstract espoused values of care into tangible care practices and communicate such practices to care staff. It is through the process of translation and communication that decisions are made regarding what is workable and acceptable in practice. For instance, empowering residents at Hebble House was translated into maintaining residents' mobility, which required staff to manage the need to keep residents safe while providing and encouraging opportunities to mobilise. Furthermore, it is important that the resulting practices are valued by senior staff members, and the potential trade-offs acknowledged (e.g. the additional time taken to perform a particular care task when enabling residents to mobilise). The ability to translate abstract values into care practice, and resolve the complexities of how it can work in practice, seem especially important with regard to promoting independence, which has been criticised for being potentially problematic in the context of health and social care for vulnerable adults and older people (Hawkins, Redley and Holland 2011 ; McCormack 2001).

This paper highlights the role of staff training and supervision, both formally and informally, in ensuring a consistent approach to care practice and 
allowing space for reflection and problem-solving. Supervision, leadership style, knowledge, skills and a solution-focused approach have been highlighted as being important in relation to high-quality care practice in care homes (Killett et al. 2015; Kirkley et al. 2011 ).

Care planning has been widely discussed in the literature, and the notion that services will provide high-quality, tailored care merely because they engage in care planning has been questioned (Mansell and Beadle-Brown 2004; McCormack 2004). Organisational factors have the potential to enable or constrain the development of high-quality care planning (McCormack 2004). This study has highlighted that living care plans that detail mobility plans for residents who require assistance to move can positively shape residents' movement during routinised activities such as moving to the dining room or bathroom. Importantly, care planning can be a mechanism for empowering care assistants to monitor and take action regarding residents' mobility and movement, and feed back important changes to seniors to obtain expert input. It is worth noting, however, that such care planning predominately shaped residents' movement during routine activities that were predictable and 'plan-able' ( $e$. $g$. walking to the dining room) and for those who required some form of support and assistance to move.

\section{Strengths and weaknesses}

This study drew on the existing notions of the care environment and organisational culture as sensitising concepts with which to consider critically indepth qualitative data on routine patterns of residents' movement in the care home context. Bringing these two concepts together to help inform the analysis is a novel approach. It enabled us to strengthen, through the inductive and deductive cycles of grounded theory analysis, our conceptualisation of the organisational element of the care home environment, allowing us to build an explanation of the organisational structures and mechanisms that shape care home residents' physical movement.

We took steps to ensure the rigour of our data collection and analysis, including keeping detailed fieldwork notes and memos, purposively sampling participants for in-depth observation and interviews, regularly meeting with the research team and study management group, and undertaking collaborative analysis.

We acknowledge the limitations of our study: we were only permitted to conduct observations in the communal areas of the participating care homes and, therefore, did not directly observe patterns of movement that occurred in private spaces such as residents' bedrooms. By supplementing the observation data with qualitative interviews we have tried to address this gap, but acknowledge that our analysis may have been limited by this. 


\section{Implications for future studies and practice}

This study has clear and significant implications for future studies, in particular those that aim to introduce interventions to increase residents' physical movement around routine activities and help maintain residents' mobility in care homes. Such studies have previously been conducted in countries in North America, Europe, Asia and Australasia (Crocker et al. 2013; Paw et al. 2008). Future research is required, to explore mechanisms for reducing sedentary behaviour during periods of leisure time when staff were not present to support and enable residents to move.

Although care homes in the UK have unique characteristics (in particular, the UK policy context and the manner in which beds are funded), existing literature from several countries (including in North America, Australasia, Europe and Asia) demonstrates that issues discussed here, such as organisational culture, leadership and management, staff training and care planning, have importance for quality of care in long-term care facilities, and aged care more generally (Jeon, Merlyn and Chenoweth 2010; Killett et al. 2015; Kirkley et al. 2011 ; Tyler and Parker 2011). This suggests the need to consider organisational culture, and the specific structures and mechanisms in place, when planning and implementing changes to increase residents' physical activity and movement in long-term care facilities in different contexts and countries.

\section{Conclusions}

Organisational structures and processes are known to shape care practices in care homes, however, the relationship between those structures and practices is nuanced and dynamic (Killett et al. 2015). By exploring the structures and processes in depth, this study has highlighted how the management processes, staff training and supervision, and care planning processes shaped residents' movement in care settings. Understanding how organisational factors shape routine movement amongst residents will inform the development of embedded and sustainable interventions that aim to enhance physical activity or reduce sedentary behaviour in care home settings.

\section{Acknowledgements}

We are extremely grateful to all the care home residents, their relatives and staff members who participated in this study. The authors would also like to thank the numerous people who assisted in the set-up and operation of the study. In particular, many thanks to Professor Anne Forster, the Chief Investigator of the programme of research; 
Nicola McMaster for her support with the project management of the study; and the members of the REACH programme management team: Dr Karen Birch, Dr David R. Ellard, Professor Amanda Farrin, Joan Firth, Professor Anne Forster, Bev Gallagher, Mary Godfrey, Elizabeth Graham, Professor Claire Hulme, Professor Rebecca Lawton, Dr Najma Siddiqi and Professor John Young for guidance received over the course of the study. We are grateful to our funders. This paper presents independent research funded by the National Institute for Health Research (NIHR) under its Programme Grants for Applied Research (PGfAR) Programme (Grant Number RP-PG-1210-12017). The views expressed are those of the authors and not necessarily those of the NHS, the NIHR or the Department of Health. NHS ethical approval was obtained for the research (Yorkshire \& The Humber Bradford Leeds Research Ethics Committee, REC Reference 12/YH/o564). RJH contributed to the design and setting up of the study, data collection, data analysis, the study management group and developed the manuscript. AP contributed to the design and setting up of the study, data collection, data analysis, the study management group and reviewed the manuscript. AL contributed to the setting up of the study, data collection, data analysis, the study management group and reviewed the manuscript. DRE is a co-applicant on the research programme grant, and contributed to the design of the study, the study management group and reviewed the manuscript. MG is a co-applicant on the research programme grant and work-stream lead, and contributed to the design and setting up of the study, data analysis, the study management group and reviewed the manuscript. The authors declare that there are no conflicts of interest.

\section{References}

Atkinson, P., Coffey, A., Delamont, S., Loftland, J. and Loftland, L. 2001. Handbook of Ethnography. Sage, London.

Baum, E. E., Jarjoura, D., Polen, A. E., Faur, D. and Rutecki, G. 2003. Effectiveness of a group exercise program in a long-term care facility: a randomized pilot trial. Journal of the American Medical Directors Association, 4, 2, 74-80.

Blake, H., Mo, P., Malik, S. and Thomas, S. 2009. How effective are physical activity interventions for alleviating depressive symptoms in older people? A systematic review. Clinical Rehabilitation, 23, $10,873-87$.

Blumer, H. 1986. Symbolic Interactionism: Perspective and Method. University of California Press, London.

Bruunsgaard, H., Bjerregaard, E., Schroll, M. and Pedersen, B. K. 2004. Muscle strength after resistance training is inversely correlated with baseline levels of soluble tumor necrosis factor receptors in the oldest old. Journal of the American Geriatrics Society, 52, 2, 237-41.

Care Quality Commission 2016. The State of Healthcare and Adult Social Care in England 2015/16. Williams Lea Group on behalf of the controller of Her Majesty's Stationary Office, London, UK.

Charmaz, K. 20oo. Grounded theory: objectivist and constructivist methods. In Denzin, N. and Lincoln, Y. (eds), Handbook of Qualitative Research. Sage, London, 509-36.

Charmaz, K. 2014. Constructing Grounded Theory. Sage, London.

Charmaz, K. and Mitchell, R.G. 2001. Grounded theory in ethnography. In Atkinson, P., Coffey, A., Delamont, S., Loftland, J. and Loftland, L. (eds), Handbook of Ethnography. Sage, London, 160-74. 
Crocker, T., Forster, A., Young, J., Brown, L., Ozer, S., Smith, J., Green, J., Hardy, J., Burns, E., Glidewell, E. and Greenwood, D. 2013 . Physical rehabilitation for older people in long-term care. Cochrane Database of Systematic Reviews, Article CDoo4294.

Gubrium, J.F. 1978. Notes on the social organization of senility. Journal of Contemporary Ethnography, 7, 1, 23-44.

Harmer, B.J. and Orrell, M. 2008. What is meaningful activity for people with dementia living in care homes? A comparison of the views of older people with dementia, staff and family carers. Aging and Mental Health, 12, 5, 54 ${ }^{8-58 .}$

Hawkins, R., Redley, M. and Holland, A. 2011 . Duty of care and autonomy: how support workers managed the tension between protecting service users from risk and promoting their independence in a specialist group home. Journal of Intellectual Disability Research, 55, 9, 873-84.

Hughes, C. M., Lapane, K., Watson, M. C. and Davies, H. T. 2007. Does organisational culture influence prescribing in care homes for older people? Drugs $\mathcal{E}$ Aging, 24, 2, 81-93.

Jeon, Y. H., Merlyn, T. and Chenoweth, L. 2010. Leadership and management in the aged care sector: a narrative synthesis. Australasian Journal on Ageing, 29, 2, 54-6o.

Katzmarzyk, P. T. 2010. Physical activity, sedentary behavior, and health: paradigm paralysis or paradigm shift? Diabetes, 59, 11, 2717-25.

Kerse, N., Peri, K., Robinson, E., Wilkinson, T., Von Randow, M., Kiata, L., Parsons, J., Latham, N., Parsons, M. and Willingale, J. 2008. Does a functional activity programme improve function, quality of life, and falls for residents in long term care? Cluster randomised controlled trial. BMJ, 337, a1 445 .

Killett, A., Burns, D., Kelly, F., Brooker, D., Bowes, A., La Fontaine, J., Latham, I., Wilson, M. and O'Neill, M. 2015. Digging deep: how organisational culture affects care home residents' experiences. Ageing $\mathcal{E}$ Society, 36, 1, 16o-88.

Kirkley, C., Bamford, C., Poole, M., Arksey, H., Hughes, J. and Bond, J. 2011. The impact of organisational culture on the delivery of person-centred care in services providing respite care and short breaks for people with dementia. Health $\mathcal{E}$ Social Care in the Community, 19, 4, 438-48.

Mansell, J. and Beadle-Brown, J. 2004. Person-centred planning or person-centred action? Policy and practice in intellectual disability services. Journal of Applied Research in Intellectual Disabilities, 17, 1, 1-9.

Matei, R., Thuné-Boyle, I., Hamer, M., Iliffe, S., Fox, K. R., Jefferis, B. J. and Gardner, B. 2015. Acceptability of a theory-based sedentary behaviour reduction intervention for older adults ('On Your Feet to Earn Your Seat'). BMC Public Health, 15, 606.

McCormack, B. 2001. Negotiating Partnerships with Older People: A Person Centred Approach. Ashgate, Aldershot, UK.

McCormack, B. 2004. Person-centredness in gerontological nursing: an overview of the literature. Journal of Clinical Nursing, 13, s1, 31-8.

Moore, K. D. 1999. Dissonance in the dining room: a study of social interaction in a special care unit. Qualitative Health Research, 9, 1, 133-55.

Moore, K. D. 2014. An ecological framework of place: situating environmental gerontology within a life course perspective. International Journal of Aging and Human Development, 79, 3, 183-209.

Owen, N., Healy, G. N., Matthews, C. E. and Dunstan, D. W. 2010. Too much sitting: the population-health science of sedentary behavior. Exercise and Sport Sciences Reviews, 38, 3, $105^{-13}$.

Paw, M.J. C. A., Chin, A., Van Uffelen, J. G., Riphagen, I. and Van Mechelen, W. 2008. The functional effects of physical exercise training in frail older people. Sports Medicine, 38, 9, 781-93. 
Potter, R., Ellard, D., Rees, K. and Thorogood, M. 2011 . Asystematic review of the effects of physical activity on physical functioning, quality of life and depression in older people with dementia. International Journal of Geriatric Psychiatry, 26, 10, 1000-1 1.

Reid, N., Eakin, E., Henwood, T., Keogh, J. W., Senior, H. E., Gardiner, P. A., Winkler, E. and Healy, G. N. 2013. Objectively measured activity patterns among adults in residential aged care. International Joumal of Environmental Research and Public Health, 10, 12, 6783-98.

Rowles, G. D. and Bernard, M. A. 2013. Environmental Gerontology: Making Meaningful Places in Old Age. Springer, New York.

Sackley, C., Hoppitt, T., Levin, S. and Cardoso, K. 2006. Observations of activity levels and social interaction in a residential care setting. International Journal of Therapy $\mathcal{E}^{\circ}$ Rehabilitation, 13, 8, 370-3.

Sardinha, L. B., Santos, D. A., Silva, A. M., Baptista, F. and Owen, N. 2015 . Breakingup sedentary time is associated with physical function in older adults. Journals of Gerontology: Biological Sciences and Medical Sciences, 7oA, 1, 119-24.

Schein, E. 1990. Organisational culture. American Psychologist, 45, 2, 109-19.

Smalbrugge, M., Pot, A. M., Jongenelis, L., Gundy, C. M., Beekman, A.T. and Eefsting, J. A. 2006. The impact of depression and anxiety on well being, disability and use of health care services in nursing home patients. International Journal of Geriatric Psychiatry, 21, 4, 325-32.

Spradley, J. P. 1980. Participant Observation. Holt, Rinehart and Winston, New York.

Thorp, A. A., Owen, N., Neuhaus, M. and Dunstan, D. W. 201 1. Sedentary behaviors and subsequent health outcomes in adults: a systematic review of longitudinal studies, 1996-2011. American Journal of Preventive Medicine, 41, 2, 207-15.

Tyler, D. A. and Parker, V.A. 2011. Nursing home culture, teamwork, and culture change. Journal of Research in Nursing, 16, 1, 37-49.

Van Alphen, H. J., Volkers, K. M., Blankevoort, C. G., Scherder, E. J., Hortobágyi, T. and Van Heuvelen, M.J. 2016. Older adults with dementia are sedentary for most of the day. PLOS ONE, 11, 3, eo1 52457.

Van Maanen, J. and Schein, E. 1979. Toward a theory of organizational socialization. In Staw, B.M. and Cummings, L.L. (eds), Research in Organizational Behavior, (Vol. I). CT : JAI Press, Greenwich, 209-69.

Windle, G., Hughes, D., Linck, P., Russell, I. and Woods, B. 2010. Is exercise effective in promoting mental well-being in older age? A systematic review. Aging $\mathcal{E}$ Mental Health, 14, 6, 652-69.

Accepted Io February 2017 ; first published online 3 I March 2017

Address for correspondence:

Rebecca Hawkins,

Academic Unit of Elderly Care and Rehabilitation,

Temple Bank House (Ground Floor),

Bradford Royal Infirmary, Duckworth Lane,

Bradford, UK BDg 6RJ

E-mail: r.hawkins@leeds.ac.uk 